\title{
BMJ Open SheppHeartCABG trial-comprehensive early rehabilitation after coronary artery bypass grafting: a protocol for a randomised clinical trial
}

\author{
Ida Elisabeth Højskov, ${ }^{1}$ Philip Moons, ${ }^{2,3}$ Niels Viggo Hansen, ${ }^{4}$ Søren La Cour, ${ }^{5}$ \\ Peter Skov Olsen, ${ }^{1}$ Christian Gluud, ${ }^{6}$ Per Winkel, ${ }^{6}$ Jane Lindschou, ${ }^{6}$ \\ Lau Caspar Thygesen, ${ }^{7}$ Ingrid Egerod, ${ }^{8}$ Selina Kikkenborg Berg ${ }^{1}$
}

To cite: Højskov IE, Moons P, Hansen NV, et al. SheppHeartCABG trialcomprehensive early rehabilitation after coronary artery bypass grafting: a protocol for a randomised clinical trial. BMJ Open 2017;7:e013038.

doi:10.1136/bmjopen-2016013038

- Prepublication history for this paper is available online. To view these files please visit the journal online (http://dx.doi.org/10.1136/ bmjopen-2016-013038).

Received 14 June 2016 Revised 12 December 2016 Accepted 14 December 2016

CrossMark

For numbered affiliations see end of article.

Correspondence to Ida Elisabeth Højskov; ida.elisabeth.hoejskov@ regionh.dk

\begin{abstract}
Introduction: Patients undergoing coronary artery bypass graft surgery often experience a range of symptoms. Studies indicate that non-pharmacological interventions such as exercise training and psychoeducation have a positive physiological and psychological effect in early outpatient rehabilitation. The SheppHeartCABG trial will investigate the effect of early comprehensive rehabilitation in early phase rehabilitation versus usual care. The aim of this paper is to present the protocol for the SheppHeartCABG trial.
\end{abstract}

Methods/analysis: SheppHeartCABG is an investigatorinitiated randomised clinical superiority trial with blinded outcome assessment, employing 1:1 central randomisation to rehabilitation plus usual care versus usual care alone. On the basis of a sample size calculation, 326 patients undergoing coronary artery bypass grafting will be included from two clinical sites. All patients receive usual care and patients allocated to the experimental intervention follow 4 weeks rehabilitation consisting of an exercise programme, psycho-educative consultations and a compact mindfulness programme. The primary outcome is physical function measured by the 6-min walk test. The secondary outcomes are mental health and physical activity measured by the Medical Outcome Study Short Form (SF-12), anxiety and depression measured by the Hospital Anxiety and Depression Scale questionnaire, physical, emotional and global scores by the HeartQoL questionnaire, sleep measured by the Pittsburgh Sleep Quality Index, pain measured by the Örebro Musculoskeletal Screening Questionnaire and muscle endurance measured by the sitto-stand test. A number of explorative analyses will also be conducted.

Ethics and dissemination: SheppHeartCABG is approved by the regional ethics committee (no. H-42014-109) and the Danish Data Protection Agency (no. 30-1309) and is performed in accordance with good clinical practice and the Declaration of Helsinki in its latest form. Positive, neutral and negative results of the trial will be submitted to international peer-reviewed journals. Furthermore, results will be presented at national and international conferences relevant to the subject fields. Trial registration number: NCT02290262; pre-results.

\section{Strengths and limitations of this study}

- This study has been designed to meet the criteria for high quality in a non-pharmacological randomised clinical trial with computergenerated randomisation, multicentre participation and blinded outcome assessment and analysis.

- We are aware of the subjective nature of the selfreported secondary outcomes. Accordingly, we will interpret data conservatively.

- This trial is the first larger trial testing the effects of a phase I comprehensive cardiac rehabilitation programme after coronary artery bypass graft surgery.

\section{BACKGROUND}

Following coronary artery bypass grafting $(\mathrm{CABG})$, patients often experience a range of symptoms caused by the surgical procedure and the ischaemic heart disease and the subsequently return to everyday life is often prolonged. ${ }^{1}$ Symptoms of anxiety and depression peak before heart surgery and again 2 weeks after and may persist up to 4 months after discharge. ${ }^{2}$ Pain, fatigue and sleep disorders are common symptoms after CABG and may be partly due to the lack of postoperative physical activity. ${ }^{1}{ }^{3}{ }^{4}$ To tackle these issues, cardiac rehabilitation is recommended. $^{5}$

Cardiac rehabilitation programmes are generally described according to three main phases: inpatient (phase I), early outpatient (phase II) and long-term outpatient cardiac rehabilitation (phase III). ${ }^{6}$ Rehabilitation phase I normally ends 4 weeks following CABG and ought to be followed by rehabilitation phase II. $^{6}$ Enrolment in cardiac rehabilitation after coronary revascularisation positively impacts the psychological status of 
patients and reduces cardiovascular mortality. ${ }^{7}$ More specifically, physical exercise in rehabilitation phase II has positive effects on quality of life, exercise capacity, coronary blood vessels, the myocardium, the endothelial function and coagulation. ${ }^{8-13}$ There are no randomised clinical trials assessing cardiac rehabilitation programmes in phase I.

\section{Non-pharmacological treatment potential}

Open heart surgery is a severe physical and mental strain. Indeed, cardiopulmonary bypass has a temporary negative effect on physical functions. ${ }^{8}{ }^{9}$ However, studies show that psychosocial factors are also vital for patients' prognosis in the short and longer term. ${ }^{10}$ The interventions in phase I rehabilitation after CABG have either investigated the physical or the psychological perspective. The effect of respiratory exercise preoperatively and in the early postoperative period after CABG surgery has been examined using different techniques. ${ }^{11-14}$ Trials generally indicate a clinically significant effect of respiratory physiotherapy, but these trials are small and include selected trial populations. Cardiopulmonary bypass has a temporary negative effect on the physical function level. ${ }^{8}{ }^{9}$ Examination of anxiety and depression in the postoperative period is needed due to the limited power and the use of non-validated instruments in the studies. ${ }^{2}$ Furthermore, it has been shown that a combination of a psycho-educative intervention plus physical exercise has a positive effect on physical and mental self-related health. ${ }^{15}{ }^{16}$ Mindfulness is a form of psychosocial intervention that offers stress reduction, calm accepting awareness and support of self-care through meditationbased exercises. Until now, trials of mindfulness have not been conducted in the context of CABG patients but several trials with cardiovascular patients in other contexts have shown reduction of perceived stress, pain, depression, sleeping problems and anxiety. ${ }^{17-20}$

International guidelines recommend early mobilisation during hospitalisation, outpatient exercise training and patient education after CABG. ${ }^{21}$ Since the symptoms related to CABG include a physical and a psychological component, it is plausible that patients with CABG benefit from a comprehensive rehabilitation intervention. ${ }^{22}$ The SheppHeartCABG pilot trial was initiated in 2013 to test the safety and tolerability of a comprehensive intervention and to provide outcome data for designing a larger trial. ${ }^{23}$ The pilot trial included 60 patients and indicated the feasibility of physical and psycho-educational cardiac rehabilitation in addition to sufficient inclusion rate and high compliance with most elements. ${ }^{23}$ The results were used to modify the rehabilitation programme and optimise the protocol and targeted outcomes.

In summary, there is evidence that physical exercise benefits heart patients' rehabilitation associated with open heart surgery. This part normally begins $2-4$ weeks after discharge, and the effects of early action are not known. In addition, the positive effect of rehabilitation may be stronger when physical exercise is combined with a psychological intervention component.

\section{TRIAL OBJECTIVES}

The objective of the SheppHeartCABG trial is to investigate the benefits and harms of a phase I comprehensive cardiac rehabilitation programme consisting of an exercise training component and a psycho-educative component, in addition to usual treatment in patients with CABG. The primary hypothesis is that a comprehensive rehabilitation programme improves the functional level measured by a 6 -min walk test (6MWT) by $30 \mathrm{~m}$ in the experimental group compared with the control group. The estimated increase in the primary outcome is a conservatively expected estimate based on the SheppHeartCABG pilot trial, where we found a mean of $548 \mathrm{~m}$ in the intervention group receiving physical training and psycho-education versus a mean of $451 \mathrm{~m}$ in the control group measured by 6MWT. ${ }^{23}$ The secondary hypotheses are that a comprehensive rehabilitation programme will improve: quality of life, sleep disorder, pain, anxiety, depression and leg strength and endurance. Exploratory analyses will evaluate whether the intervention will have a positive impact on: fatigue, physical activity, cognitive and emotional representation of illness and self-rated health.

\section{METHODS}

SheppHeartCABG is an investigator-initiated randomised clinical superiority trial with a blinded outcome assessment, employing 1:1 randomisation to a comprehensive cardiac rehabilitation programme plus usual care versus usual care alone. Patients will be recruited from two university hospitals in Denmark. Figure 1 shows the trial design.

\section{Trial population and eligibility criteria}

Patients aged 18 years and older admitted for elective CABG who speak and understand Danish, and provide written informed content, are considered eligible for participation. Exclusion criteria are patients at intermediate or high risk in relation to their cardiovascular status $^{24}$ and patients with orthopaedic conditions that would prohibit walking and cycling exercise.

\section{Experimental intervention}

The experimental intervention is a comprehensive rehabilitation programme with an exercise training component and a psycho-educational component from admission until 4 weeks after CABG surgery.

\section{The physical components}

The exercise programme during hospitalisation consists of respiratory physiotherapy, neck and shoulder exercises, walking and cycling. The purpose is preventing respiratory complications such as atelectasis and pneumonia, neck and shoulder pain and increasing the 


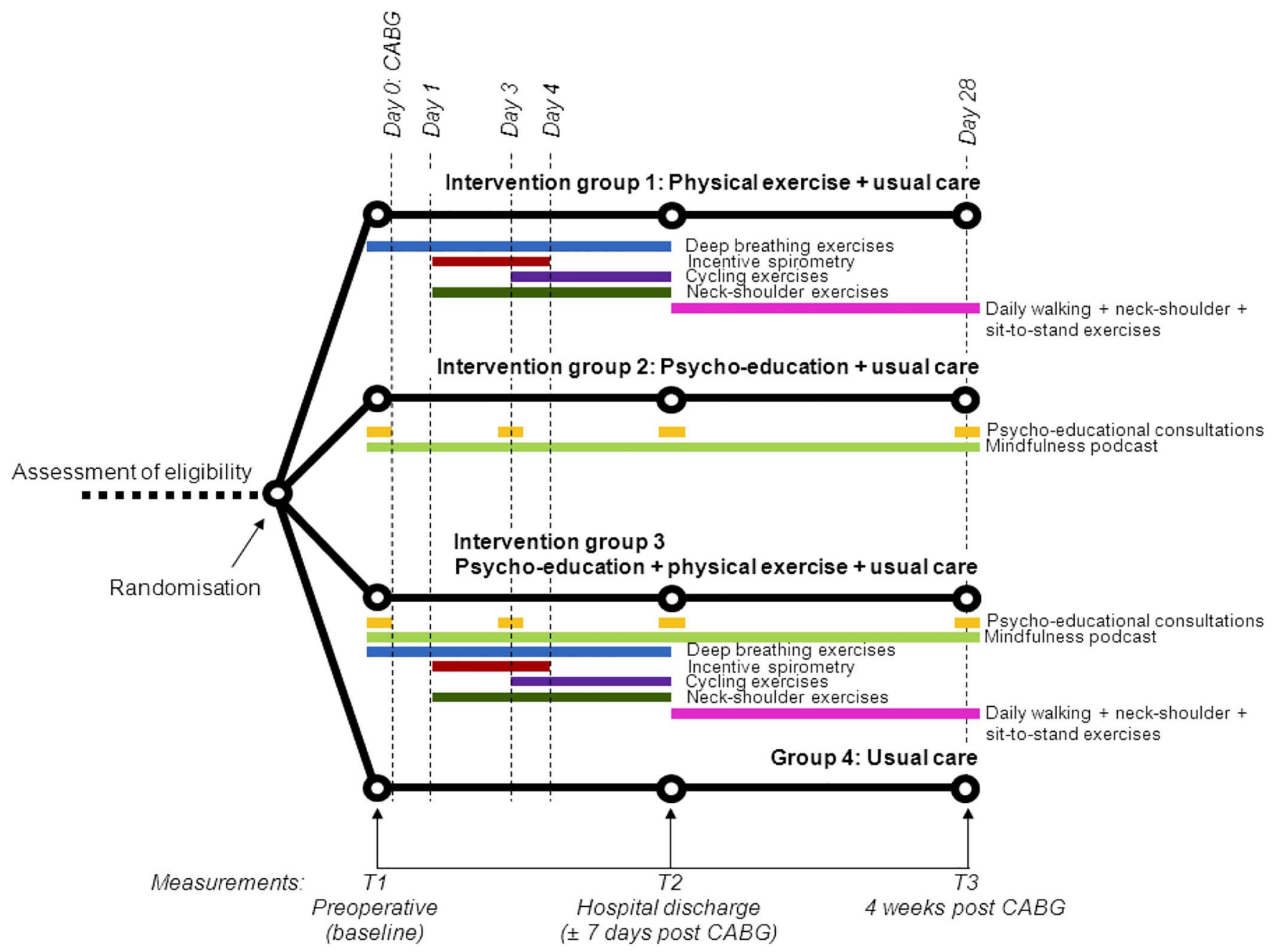

\begin{tabular}{|c|c|c|}
\hline & & \\
\hline $\begin{array}{l}\text { Patient-reported outcomes } \\
\text { - } \text { SF-36 } \\
\text { - HeartQoL } \\
\text { - } \text { HADS } \\
\text { - } \text { MFI-2O } \\
\text { : } I P A Q \\
\text { - } \text { B-IPQ } \\
\text { - } \text { PSQI } \\
\text { OMPSQ }\end{array}$ & $\begin{array}{l}\text { Functional capacity } \\
\text { - Cardiopulmonary } \\
\text { exercise testing } \\
\text { - } 6 \text { minutes' walk test } \\
\text { - Sit and stand test } \\
\text { Patient-reported outcomes } \\
\text { - SF-36 } \\
\text { - HADS } \\
\text { - B-IPQ } \\
\text { - OMPSQ }\end{array}$ & $\begin{array}{l}\text { Functional capacity } \\
\text { - Cardiopulmonary } \\
\text { exercise testing } \\
\text { - } 6 \text { minutes' walk test } \\
\text { - Sit and stand test } \\
\text { Patient-reported outcomes } \\
\text { - SF-36 } \\
\text { - HeartQoL } \\
\text { - HADS } \\
\text { - MFI-20 }\end{array}$ \\
\hline & & $\begin{array}{l}\text { - } B-I P Q \\
\text { - } P S Q I \\
\text { - ÖMPSQ }\end{array}$ \\
\hline
\end{tabular}

Figure 1 Trial design. CABG, coronary artery bypass grafting; HADS, Hospital Anxiety and Depression Scale; NYHA, New York Heart Association; PSQI, Pittsburgh Sleep Quality Index.

physical functional level. Each exercise in the training programme is based on guidelines, ${ }^{25}$ public health recommendations for physical activity for adults ${ }^{26}$ and supported by European recommendations for physical training in patients with cardiac disease. ${ }^{21} 27$ Physical exercise starts immediately after surgery and follows the ACSM's Guidelines for Exercise Testing and Prescription. ${ }^{25}$ A physiotherapist with specific knowledge of open heart surgery and cardiac rehabilitation initiates the programme and gives the participants standardised 
instructions in each part of the programme. All the completions of exercises are documented in the training diary.

\section{Physical exercises during hospitalisation Breathing exercise}

This consists of 7-10 deep breaths four times each day when awake in daytime. Begin at admission and continue until hospital discharge.

\section{Peak flow spirometry}

Participants are encouraged to perform breathing exercises consisting of 3-5 min of breathing in the PEP-flute four times daily during postoperative days 1 through 4 .

\section{Walking}

Walking exercises are performed in the hallway at the hospital ward on postoperative day 1 continuing to 4 weeks postsurgery. Walking exercise begins on the first postoperative day with a $2 \times 5$ min walk; second postoperative day a $2 \times 7$ min walk; third postoperative day a $3 \times 7$ min walk and from the fourth postoperative day until discharge a $3 \times 10$ min walk. The participant has to increase the intensity of walking from low to moderate during hospital admission.

\section{Neck and shoulder}

This consists of shoulder lifts, shoulder rolls and head rolls with 10 sessions of each exercise two times per day from postoperative day 1 until hospital discharge.

\section{Cycling}

Cycling exercise training is conducted on stationary bicycles or alternatively using bed bikes. ${ }^{28}$ Heart rate and saturation are monitored at three scheduled times during the two first exercise sessions. The participants are encouraged to train using moderate intensity, so that they are not exhausted and the training is safe and has effect. ${ }^{29}$ Time for cycling will be $10 \mathrm{~min}$. The rating of perceived exertion scales (RPE) is used to measure the exercise intensity. The cycling exercise intensity is at RPE Borg between 13 and 15 on a scale of $6-20,{ }^{30}$ and the power on the cycle is programmed so that it matches this intensity. There will be a 5 min warm-up and a 5 min cool-down to achieve cardiovascular adjustment with exercise intensity $\leq 10$ RPE Borg. ${ }^{31}{ }^{32}$ The cycling exercise consists of two daily sessions from the third postoperative day until discharge.

\section{Physical exercise from discharge to 4 weeks after surgery}

Exercise training will take place at the participants' home and outdoors including resistance training and walking to obtain muscle strength and physical capacity. ${ }^{33}$ At hospital, the physiotherapist introduces the exercises and the training diary.

\section{Walking}

The length of daily walk will be increased from hospital discharge until 4 weeks as follows: $3 \times 10 \mathrm{~min}, 2 \times 15 \mathrm{~min}$, $2 \times 20 \mathrm{~min}, 2 \times 25 \mathrm{~min}$ and $2 \times 30 \mathrm{~min}$. The walking intensity is moderate: the first $2-3$ min $\leq 10-13$ on the Borg Scale and the last 2-3 $\mathrm{min}$ at the same intensity level and the time between 12 and 14 on the Borg Scale.

\section{Leg endurance and strength}

The participants perform a sit and stand exercise and 'up on toes' with 10 repetitions two times per day from hospital discharge until 4 weeks after CABG.

\section{The psycho-educational components}

The interventions consist of four consultations: admission day, second postoperative day, discharge and 3 weeks after CABG. The first three consultations are in-hospital, and the fourth is by phone. The psycho-educative intervention has two parts: one inspired by the three dimensions in Rosemarie R Parse's 'Human Becoming Practice Methodologies' ${ }^{34}$ and the second is a compact mindfulness programme combing dialogue and recorded instructions for individual practice. ${ }^{35}$ The overall goal of the psycho-educative intervention is that the patients learn to construe and react to relevant physical and psychological symptoms and thus learn to cope with anxiety and fear after CABG.

\section{Consultations}

Four consultations are scheduled: admission day, second postoperative day, discharge and 3 weeks after CABG. The first three consultations are in-hospital, and the fourth is by phone. The consultations are conducted by specially trained nurses and last for about $45 \mathrm{~min}$. Rosemarie R Parse's 'Human Becoming Practice Methodologies ${ }^{, 34}$ forms the conceptual foundation for the dialogues with the patients.

The Human becoming Practice Method describes three dimensions for dialogue: (1) discuss and give meaning to the past, present and future; (2) explore and discuss events and opportunities and (3) pursue imagined possibilities. According to this method, there are three ways to make health changes: (1) using creative images to explore, (2) recognising personal patterns and (3) value priorities and shed light on the paradoxes by looking at the incongruence in a situation and change the view of reality. The nurse is 'truly present' in the process through discussion, silent immersion and reflection.

\section{Mindfulness}

The other part of the psycho-educational intervention is a set of mindfulness exercises that patients can use $a d$ libitum for stress reduction, calm awareness and self-care through meditation-based exercises. ${ }^{17}{ }^{36}$ For this trial, a brief mindfulness intervention with several components has been developed in cooperation with the Center for Research in Existence and Society, University of 
Copenhagen. It particularly focuses on the patients with CABG in need of calming down in physically and emotionally stressful situations. At the first consultation, participants are introduced to mindfulness through a short mindfulness exercise lead by the nurse, followed by a dialogue. The participants receive an $\mathrm{mp}-3$ player with an audio mindfulness programme. The audio mindfulness programme consists of three guided meditations of $5 \mathrm{~min}, 15 \mathrm{~min}$ and $20 \mathrm{~min}$, and the participants are encouraged to listen to all three meditations from admission to 4 weeks after CABG.

\section{Usual care}

Patient in both arms of the trial receive usual care according to the guidelines. ${ }^{6} 37$ The standard admission time after CABG is 5-8 days. Usual treatment involves preoperative and postoperative information provided by physicians, nurses and physiotherapists. Instructions regarding precautions after sternotomy are provided by physiotherapists covering the immediate postoperative period during hospitalisation and after discharge. Primary perioperative nursing consists of admission interview with preoperative screening (falls, nutrition); introduction to postoperative pain and nausea medications; pain assessment and postoperative activities. Early postoperative care focuses on the observation of vital signs, while the remainder of the hospital admission focuses on recovery and preparation for discharge.

\section{Outcomes and data collection}

Data will be collected at admission, discharge and 4 weeks following surgery (see table 1 ).

\section{Primary outcome}

The primary outcome in this trial is physical functional level as measured by the 6MWT. 6MWT is a simple tool and will be administered at admission, discharge and 4 weeks following CABG. ${ }^{38}$ For the $6 \mathrm{MWT}$, the participants walked up and down a $30 \mathrm{~m}$ hallway for $6 \mathrm{~min}$ according to the guidelines. ${ }^{39}$ To ensure standard testing, a standardised instruction for patients has been developed.

\section{Secondary outcomes}

Furthermore, we will assess nine preplanned secondary hypotheses based on estimates using the SDs from the SheppHeartCABG pilot trial. ${ }^{23}$ The variables, instruments, subscales, Cronbach's $\alpha$ and hypothesised differences are detailed in table 2.

\section{The Medical Outcome Study Short Form 12 (SF-12)}

Mental health and physical health are measured by the Medical Outcome Study Short Form 12 (SF-12) 4 weeks after surgery. The SF-12 is a 12-item validated version of the SF-36 and is a brief, reliable measure of overall health status that generates a physical component score and a mental component score (PCS and MCS). ${ }^{40}$

\section{The Hospital Anxiety and Depression Scale}

This 14-item instrument measures symptoms of anxiety and depression ${ }^{41}{ }^{42}$ The scale offers two scores HADS-A and HADS-D, each of which can range from 0 to 21. Scores of $0-7$ for either subscale are regarded as normal; 8-10 suggest the presence of a mood disorder and 11 and above suggest the probable presence of a mood disorder.

\section{The HeartQol Questionnaire}

Health-related quality of life is measured with the use of the HeartQol questionnaire. ${ }^{43}$ The emotional subscale and the global scale will be included which are scored from 0 to 3 . The questionnaire consists of 14 items.

Table 1 SheppHeartCABG: demographic and baseline characteristics, tests and questionnaires

\begin{tabular}{|c|c|c|}
\hline Quantity & $\begin{array}{l}\text { Time of } \\
\text { measure }\end{array}$ & $\begin{array}{l}\text { Type of } \\
\text { quantity }\end{array}$ \\
\hline \multicolumn{3}{|l|}{ Demographic } \\
\hline Age, height, weight, martial, educational & Baseline & Continuous \\
\hline Occupational status & Baseline & Categorical \\
\hline Smoking & Baseline & Binary $(\mathrm{Y} / \mathrm{N})$ \\
\hline \multicolumn{3}{|l|}{ Clinical } \\
\hline Nutritional status (BMI) & Baseline & Continuous \\
\hline NYHA calcification & Baseline & Continuous \\
\hline Type of heart disease & Baseline & Categorical \\
\hline Diabetes mellitus & Baseline & Binary $(\mathrm{Y} / \mathrm{N})$ \\
\hline Medical status & Baseline & Categorical \\
\hline Level of physical activity & Baseline, discharge, W4 & Continuous \\
\hline \multicolumn{3}{|l|}{ Functional level } \\
\hline 6MWT and Sit and stand test & Baseline, discharge, W4 & Continuous \\
\hline \multicolumn{3}{|l|}{ Questionnaire } \\
\hline SF 12, HADS, ÖMPSQ, B-IPQ, EQ-5D & Baseline, discharge, W4 & Continuous \\
\hline HeartQol, PSQI, MFI-20, IPAQ & Baseline, W4 & Continuous \\
\hline
\end{tabular}


Table 2 Overview of secondary outcomes

\begin{tabular}{|c|c|c|c|c|}
\hline Variables & Instrument & Subscales & $\begin{array}{l}\text { Cronbach's } \\
\text { alpha }\end{array}$ & $\begin{array}{l}\text { Hypothesised difference } \\
\text { (anticipated power) }\end{array}$ \\
\hline Health status & $\begin{array}{l}\text { Medical Outcome Study Short } \\
\text { Form } 12 \text { (SF-12) }\end{array}$ & $\begin{array}{l}\text { Physical Component } \\
\text { Summary (PCS) [0-100] } \\
\text { Mental Component } \\
\text { Summary (MCS) [0-100] }\end{array}$ & $\begin{array}{l}0.87 \\
0.84\end{array}$ & $\begin{array}{l}2.4(97 \%) \\
8.21(100 \%)\end{array}$ \\
\hline $\begin{array}{l}\text { Anxiety- } \\
\text { depression } \\
\text { Quality of life }\end{array}$ & $\begin{array}{l}\text { Hospital Anxiety and } \\
\text { Depression Scale (HADS) }{ }^{41} 42 \\
\text { HeartQol questionnaire }^{4357}\end{array}$ & $\begin{array}{l}\text { HADS-A scale }[0-21] \\
\text { HADS-D scale }[0-21] \\
\text { The emotional subscales } \\
\text { [0-3] } \\
\text { The global subscales [0-3] }\end{array}$ & $\begin{array}{l}0.83 \\
0.82 \\
0.80-0.91 \\
0.80-0.91\end{array}$ & $\begin{array}{l}1.26(86 \%) \\
4.3(100 \%) \\
0.3(85 \%) \\
0.2(95 \%)\end{array}$ \\
\hline Sleep & $\begin{array}{l}\text { Pittsburgh Sleep Quality Index } \\
\text { (PSQI) }^{58}\end{array}$ & $\begin{array}{l}\text { Component 1: Subjective } \\
\text { sleep quality score [0-3] } \\
\text { Component 2: Sleep } \\
\text { latency score [0-3] } \\
\text { Component 3: Sleep } \\
\text { duration score [0-3] } \\
\text { Component 4: Habitual } \\
\text { sleep efficiency score [0-3] } \\
\text { Component 5: Step } \\
\text { disturbances score[0-3] } \\
\text { Component 6: Use of } \\
\text { sleeping medication score } \\
\text { [0-3] } \\
\text { Component 7: Daytime } \\
\text { dysfunction score [0-3] } \\
\text { Global PQSI score [0-21] }\end{array}$ & 0.83 & $2.2(96 \%)$ \\
\hline Pain & $\begin{array}{l}\text { Örebro Musculoskeletal Pain } \\
\text { Questionnaire (OMPQ) }\end{array}$ & OMPQ total score [0-210] & 0.86 & $10.2(98 \%)$ \\
\hline $\begin{array}{l}\text { Strength and } \\
\text { endurance in legs }\end{array}$ & Sit-to-stand test ${ }^{39}$ & Total number of repetitions & NR & $3.6(100 \%)$ \\
\hline
\end{tabular}

\section{The Pittsburgh Sleep Quality Index}

This is a self-rated questionnaire which assesses quality and disturbances of sleep over a 1-month time interval. Nineteen items generate seven component scores. The sum of scores for these seven components yields one global score.

\section{The Örebro Musculoskeletal Screening Questionnaire}

Pain is measured by the Örebro Musculoskeletal Screening Questionnaire. This 25-item self-administered questionnaire is formulated such that all musculoskeletal pains are addressed and assessed in five categories. ${ }^{44}$

\section{Sit-to-stand test}

Strength and endurance in legs is measured by a sit-to-stand test 4 weeks following surgery. ${ }^{45}$ The test is carried out using a chair. The participants are then seated on the chair and have to get up to full standing position and then return to the initial seated position as many times as possible in $30 \mathrm{~s}$. The test will be performed in accordance with guidelines. ${ }^{46}$

\section{Exploratory outcomes}

Clinical and demographic data will be evaluated. The HeartQol physical component will be analysed as an exploratory outcome. ${ }^{43}$ Furthermore, a series of questionnaires regarding fatigue, physical activity and perception of illness are administered. The Measurement of Fatigue Instrument ${ }^{47-50}$ is a 20 item self-report instrument designed to measure fatigue. The International Physical Activity Questionnaire ${ }^{51}$ is used to measure health-related physical activity. Perception of illness is measured by The Brief perception Questionnaire, ${ }^{52}$ which is a short questionnaire that assesses cognitive and emotional representations of illness on the basis of eight items. The EQ-5D is a standardised instrument for use as a measure of current health status that provides a simple descriptive profile and a single index value that can be used in clinical and economic evaluation of healthcare and in population health surveys. ${ }^{53}$

\section{Sample size and power calculations}

The study is a randomised superiority trial with the continuous outcome 6MWT with 1 control per experimental participant. In the pilot trial, the outcome was normally distributed with an SD of $90 \mathrm{~m}$. If the true difference in the experimental and control means is $30 \mathrm{~m}$, the trial shall include 163 experimental participants and 163 control participants (total participants 326) to be 
able to reject the null hypothesis that the population means of the experimental and control groups are equal with probability (power) $85 \%$. The type I error probability associated with this test of this null hypothesis is $5 \%$.

Based on SheppHeartCABG the pilot trial, several of the secondary outcomes are overpowered as shown previously. For all outcomes except HeartQol physical, the power to reject the null hypothesis was above $85 \%$ (type I error $5 \%$ ) (see table 2).

\section{Study procedure and randomisation}

To achieve our estimated sample size of 326 participants, patients will be identified and screened consecutively from the current elective CABG waiting list. Patients similar to the New York Heart Association (NYHA) groups I-III and without physical illness which prevents physical exercise are suitable participants in the trial. Verbal information will be given to the patient on admission day and is planned so it is possible for relatives to participate by staff personal. If a patient accepts to participate after verbal and written informed consent, the patient is randomised to the rehabilitation plus usual care or usual care alone. The allocation sequence is computer generated with a varying block size concealed by the investigators. The allocation will be conducted centrally using the web-based 'Copenhagen Trial Unit Online Randomisation System', and selected relevant participant information will be registered (civil registration number (CPR), participant number and stratum). Stratification variables are Rigshospitalet, University of Copenhagen and Odense Universitetshospital, University of Southern Denmark and sex. After allocation, the investigator immediately informs the patient of the result and the further plan. For both groups, follow-up assessments including physical tests and questionnaires will take place at admission, discharge and 4 weeks following surgery. Questionnaires will be completed on paper or electronically in the questionnaire system Enalyzer. Data management is handled independently from the researchers who interpret the data. All data are stored electronically in a coded database and in an independent spreadsheet, which is only accessible by the SheppHeart staff. The recruitment process will continue until 326 patients have been included.

\section{Blinding}

It is not possible to blind the interventions to the staff and patients. However, all physical tests, data collection and data management will be conducted by staff blinded to the interventions. Statistical analyses and drawing of conclusions from these will also be conducted blinded to the intervention group.

\section{Statistical analysis}

\section{Analysis of primary and secondary outcomes}

The primary and all secondary outcomes are continuous. For all primary and secondary outcomes except
HeartQol and HADS, it is expected that the scores will be normally distributed.

Analyses will be intention-to-treat analyses using twosided tests with a significance level of $5 \%$. There are two types of comparison between the intervention groups: (1) comparison of values measured at week 4 and (2) comparison of values measured at discharge and at 4 weeks. In the first type, a general univariate linear model is used (except for HeartQol and HADS (see below)), adjusted for baseline values. In the second type (except for HeartQol and HADS (see below)), a mixed linear model including an unstructured covariance matrix is used. In this model, the interaction between intervention and time is of main interest. If assumptions of the models are not fulfilled with reasonable approximation, non-parametric sensitivity tests will be performed.

HeartQol quantities are converted to binary quantities based on the median score, and logistic regression is used to compare the two groups at 4 weeks adjusted for value at baseline. HADS is reported as mean (SD) and is converted to binary quantities expressed as probable anxiety and depression (scores $\geq 8$ ). Logistic regression is used to compare the intervention and control groups at 4 weeks adjusted for value at baseline.

Since almost all secondary outcomes are overpowered, Cohen's $\mathrm{d}$ will be calculated for each outcome to test the clinical effect. SAS V.9.3 will be used.

\section{Missing values and multiplicity}

For the primary and secondary outcomes, multiple imputation (MI) of missing values using the Markov Chain Monte Carlo (MCMC) approach will be performed if the number of incomplete participants is above $5 \%$. The variables included are the group membership, stratifying variables (site and sex), time (baseline, discharge and 4 weeks after discharge) and outcomes. If MI is used, the primary result will be that based on the multiply imputed data sets. Significant (adjusted $\mathrm{p}<0.05$ ) results of the primary and secondary outcomes will be supplemented by the following worst case sensitivity analysis. Let $\mathrm{A}$ be the group where a beneficial significant effect is observed and B be the other group. Missing values in group A will be imputed by the minimum value found in the material, and missing values in group $B$ will be imputed by the maximum value found. When the treatment code is broken, the results may be interpreted.

The primary outcome (6MWT) will first be tested using a significance level of 0.05 . Analyses of the secondary outcome measures as preplanned in the protocol will be analysed with no adjustment of $p$ values due to multiplicity. Instead, the interpretation of each secondary outcome measure will be assessed in the light of multiple testing, that is, statistically significant effects will be interpreted in the context of increased risk of type-I error. No significance testing will be performed for the explorative outcomes. 
In addition to the primary intention-to-treat analysis, we will also perform a per-protocol analysis to include information on adherence to the intervention in the intervention group. ${ }^{54}$ The per-protocol definition reflects the two components of the intervention, in that patients in the intervention group must have completed $75 \%$ per cent of the programme in the per-protocol analysis.

\section{Ethics, safety and dissemination}

The trial will be conducted according to the latest Declaration of Helsinki. It has been registered at ClinicalTrials.gov no NCT02290262 before inclusion of the first participant. Information about the trial is given verbally and in writing. Eligible patients will be enrolled as trial participants. Trial participants are free to withdraw their informed consent at any time and will be treated according to the department's standard procedures. Patients who leave the trial will be asked for permission to continue to collect data and to use already collected data. If the patient gives permission, data will be included in the final analysis. If a patient refuses the use of already collected data, all related data will be destroyed. The trial will be conducted according to Act. No. 593 of 14 June 2011 on Act on Research Ethics Review of Health Research Projects. The investigator will immediately notify the regional ethics committee if, within the interventions period, there occur serious adverse events or serious adverse reactions. An independent Data Monitoring and Safety Committee (DMSC) has been established.

\section{Safety}

Throughout the trial, annual reports including all expected or unexpected adverse events or reactions will be submitted to the ethical committee. Reports will be accompanied by an assessment of the participants' safety. The investigator notifies the committee within 90 days of completion of the trial.

\section{Dissemination plan}

Positive, neutral and negative results of the trial will be submitted to international peer-reviewed journals. Furthermore, results will be presented at national and international conferences relevant to the subject fields. Authorship will be allocated using the guidelines for authorship defined by the International Committees of Medical Journal Editors and depends on personal involvement. Ethic Committees and component authorities will be able to obtain direct access to data and documentation.

\section{DISCUSSION}

This SheppHeartCABG trial, assessing the effect of a comprehensive cardiac rehabilitation in phase I rehabilitation on a population undergoing CABG, is the first randomised clinical trial in a representative population. This trial is expected to contribute results that can improve patient outcomes related to ischaemic heart disease treated by CABG and early rehabilitation. This is the first trial to test the effect of a comprehensive rehabilitation programme in rehabilitation phase I after CABG. SheppHeartCABG has been designed to meet the criteria for high quality in a non-pharmacological clinical trial with central stratified randomisation and two centre participation which secures against selection bias. ${ }^{55} 56$

The primary outcome and all statistical analysis are blinded to intervention, which should reduce detection and interpretation bias. ${ }^{55} 56$

\section{Trajectory}

Inclusion was initiated on November 2014 and is expected to continue until July 2016.

\section{Author affiliations}

${ }^{1}$ Department of Cardiothoracic Surgery, Rigshospitalet, Copenhagen University Hospital, Copenhagen, Denmark

${ }^{2}$ KU Leuven Department of Public Health and Primary Care, KU Leuven, Leuven, Belgium

${ }^{3}$ Institute of Health and Care Sciences, University of Gothenburg, Gothenburg, Sweden

${ }^{4}$ Interacting Minds Center, Aarhus University, Aarhus, Denmark

${ }^{5}$ The Centre for Research in Existence and Society, University of Copenhagen, Copenhagen, Denmark

${ }^{6}$ Copenhagen Trial Unit, Centre for Clinical Intervention Research, Rigshospitalet, Copenhagen University Hospital, Copenhagen, Denmark ${ }^{7}$ National Institute of Public Health, University of Southern Denmark, Copenhagen, Denmark

${ }^{8}$ Department of Neuroanaesthesiology, Rigshospitalet, Copenhagen University Hospital, Copenhagen, Denmark

Acknowledgements The test and rehabilitation team responsible for the trial comprises the Departments of Occupational Therapy and Physiotherapy at Rigshospitalet and Odense Universitetshospital. The nurses responsible for the psycho-educational intervention are recruited from the Departments of Cardiothoracic Surgery. The authors thank Marianne Tewes, Lise Henriques, Helle Greve and Dorte Bæk Olsen for discussions during the protocol development.

Contributors IEH, LCT and SKB drafted the manuscript. LCT has specifically designed the statistical analysis plan. All authors designed the trial, developed the protocol, revised the manuscript critically and have given their final approval of the version to be published.

Funding The SheppHeartCABG has received funding(s) from The Heart Centre research foundation, Rigshospitalet, The foundation of Cardiothoracic Surgery, Rigshospitalet, Metropolitan University College, The Lundbeck Foundation (FP-55- 2013), Aase \& Ejnar Danielsen Foundation (10-001245), Association Oestifterne (14-105 and 16-070), Research Foundation Rigshospitalet and Odense Universitetshospital (13-A752).

Competing interests None declared.

Provenance and peer review Not commissioned; externally peer reviewed.

Open Access This is an Open Access article distributed in accordance with the Creative Commons Attribution Non Commercial (CC BY-NC 4.0) license, which permits others to distribute, remix, adapt, build upon this work noncommercially, and license their derivative works on different terms, provided the original work is properly cited and the use is non-commercial. See: http:// creativecommons.org/licenses/by-nc/4.0/

\section{REFERENCES}

1. Lie I, Bunch EH, Smeby NA, et al. Patients' experiences with symptoms and needs in the early rehabilitation phase after coronary artery bypass grafting. Eur J Cardiovasc Nurs 2012;11:14-24. 
2. Fredericks S, Lapum J, Lo J. Anxiety, depression, and selfmanagement: a systematic review. Clin Nurs Res 2012;21:411-30.

3. Morone NE, Weiner DK, Belnap BH, et al. The impact of pain and depression on recovery after coronary artery bypass grafting. Psychosom Med 2010;72:620-5.

4. Taillefer MC, Carrier M, Bélisle S, et al. Prevalence, characteristics, and predictors of chronic nonanginal postoperative pain after a cardiac operation: a cross-sectional study. J Thorac Cardiovasc Surg 2006;131:1274-80.

5. Pourafkari L, Ghaffari S, Shahamfar J, et al. The psychological effects of cardiac rehabilitation after coronary revascularization. Turk Kardiyol Dern Ars 2016;44:228-36.

6. Hillis LD, Smith PK, Anderson JL, et al. 2011 ACCF/AHA guideline for coronary artery bypass graft surgery: executive summary: a report of the American College of Cardiology Foundation/American Heart Association Task Force on Practice Guidelines. J Thorac Cardiovasc Surg 2012;143:4-34.

7. Anderson L, Oldridge N, Thompson DR, et al. Exercise-based cardiac rehabilitation for coronary heart disease: cochrane systematic review and meta-analysis. J Am Coll Cardiol 2016;67:1-12.

8. Taggart DP, el-Fiky M, Carter R, et al. Respiratory dysfunction after uncomplicated cardiopulmonary bypass. Ann Thorac Surg 1993;56:1123-8

9. van Belle AF, Wesseling GJ, Penn OC, et al. Postoperative pulmonary function abnormalities after coronary artery bypass surgery. Respir Med 1992;86:195-9.

10. Whalley B, Thompson DR, Taylor RS. Psychological interventions for coronary heart disease: cochrane systematic review and meta-analysis. Int J Behav Med 2014;21:109-21.

11. Savci S, Degirmenci B, Saglam M, et al. Short-term effects of inspiratory muscle training in coronary artery bypass graft surgery: a randomized controlled trial. Scand Cardiovasc J 2011;45:286-93.

12. Stein R, Maia CP, Silveira AD, et al. Inspiratory muscle strength as a determinant of functional capacity early after coronary artery bypass graft surgery. Arch Phys Med Rehabil 2009;90:1685-91.

13. Westerdahl E, Lindmark B, Eriksson T, et al. Deep-breathing exercises reduce atelectasis and improve pulmonary function after coronary artery bypass surgery. Chest 2005;128:3482-8.

14. Herdy AH, Marcchi PL, Vila A, et al. Pre- and postoperative cardiopulmonary rehabilitation in hospitalized patients undergoing coronary artery bypass surgery: a randomized controlled trial. Am J Phys Med Rehabil 2008;87:714-19.

15. Berg SK, Pedersen PU, Zwisler AD, et al. Comprehensive cardiac rehabilitation improves outcome for patients with implantable cardioverter defibrillator. Findings from the COPE-ICD randomised clinical trial. Eur J Cardiovasc Nurs 2015;14:34-44.

16. Berg SK. Comprehensive rehabilitation for patients with ICD $[\mathrm{PhD}$ thesis]. Aarhus University, 2011.

17. Fjorback LO, Arendt M, Ornbøl E, et al. Mindfulness-based stress reduction and mindfulness-based cognitive therapy: a systematic review of randomized controlled trials. Acta Psychiatr Scand 2011;124:102-19.

18. Praissman S. Mindfulness-based stress reduction: a literature review and clinician's guide. J Am Acad Nurse Pract 2008;20:212-16.

19. Gross CR, Kreitzer MJ, Reilly-Spong M, et al. Mindfulness-based stress reduction versus pharmacotherapy for chronic primary insomnia: a randomized controlled clinical trial. Explore (NY) 2011:7:76-87.

20. Nakamura $\mathrm{Y}$, Lipschitz DL, Kuhn R, et al. Investigating efficacy of two brief mind-body intervention programs for managing sleep disturbance in cancer survivors: a pilot randomized controlled trial. $J$ Cancer Surviv 2013;7:165-82.

21. Piepoli MF, Corra U, Benzer W, et al. Secondary prevention through cardiac rehabilitation: from knowledge to implementation. A position paper from the Cardiac Rehabilitation Section of the European Association of Cardiovascular Prevention and Rehabilitation. Eur J Cardiovasc Prev Rehabil 2010:17:1-17.

22. Balady G. Exercise Prescription for Patients with Cardiovascular and Cerebrovascular Disease. In: Precatello L, Arena R, Riebe D, et al. eds. ACSM's Guidelines for Exercise Testing and Prescription. 9th edn. Chapter 9, pp: 236-57. Wolthers Kluwer. Lippincott Williams \& Wilkins. 2013.

23. Højskov IE, Moons P, Hansen NV, et al. Early physical training and psycho-educational intervention for patients undergoing coronary artery bypass grafting. The SheppHeart randomized $2 \times 2$ factorial clinical pilot trial. Eur J Cardiovasc Nurs 2016;15:245-37.

24. Shenkman Z, Shir $Y$, Weiss YG, et al. The effects of cardiac surgery on early and late pulmonary functions. Acta Anaesthesiol Scand 1997;41:1193-9.

25. Pescatello, LS, Arena, R, Riebe D, Thompson, PD eds ACSM's Guidelines for exercise testing and prescription. 9th edn. Wolters
Kluwer, Lippincott Williams \& Wilkins: American College of Sports Medicine, 2013

26. Pedersen, BK, Andersen LB. Physical activity. Handbook in Prevention and Treatment (In Danish). National Board of Health. 2011. http://www. sst.dk/ /media/6B3A4AE698BC42139572C76C5854BA76.ashx.

27. Mezzani A, Agostoni P, Cohen-Solal A, et al. Standards for the use of cardiopulmonary exercise testing for the functional evaluation of cardiac patients: a report from the exercise physiology section of the European Association for Cardiovascular Prevention and Rehabilitation. Eur J Cardiovasc Prev Rehabil 2009;16:249-67.

28. Hirschhorn AD, Richards DA, Mungovan SF, et al. Does the mode of exercise influence recovery of functional capacity in the early postoperative period after coronary artery bypass graft surgery? a randomized controlled trial. Interact Cardiovasc Thorac Surg 2012;15:995-1003.

29. Tabet JY, Meurin P, Teboul F, et al. Determination of exercise training level in coronary artery disease patients on beta blockers. Eur J Cardiovasc Prev Rehabil 2008;15:67-72.

30. Borg GA. Psychophysical bases of perceived exertion. Med Sci Sports Exerc 1982;14:377-81.

31. Lampman RM, Knight BP. Prescribing exercise training for patients with defibrillators. Am J Phys Med Rehabil 2000;79:292-7.

32. Fitchet A, Doherty PJ, Bundy C, et al. Comprehensive cardiac rehabilitation programme for implantable cardioverter-defibrillator patients: a randomised controlled trial. Heart 2003;89:155-60.

33. Gielen S, Niebauer J, Hambrecht R. Exercise training in heart failure. In: Perk J, Gohlke H, Hellemans I. et al. eds. Cardiovascular prevention and rehabilitation. Chapter 19 pp. 142-155. Springer, 2007.

34. Parse R. The human becoming school of thought: a perspective for nurses and other health professionals. Thousand Oaks (CA): Stage, 1998.

35. Creswell JD, Irwin MR, Burklund LJ, et al. Mindfulness-based stress reduction training reduces loneliness and pro-inflammatory gene expression in older adults: a small randomized controlled trial. Brain Behav Immun 2012;26:1095-101.

36. Smith BW, Shelley BM, Dalen J, et al. A pilot study comparing the effects of mindfulness-based and cognitive-behavioural stress reduction. J Altern Complement Med 2008;14:251-8.

37. Martin CG, Turkelson SL. Nursing care of the patient undergoing coronary artery bypass grafting. J Cardiovasc Nurs 2006;21:109-17.

38. Solway S, Brooks D, Lacasse $\mathrm{Y}$, et al. A qualitative systematic overview of the measurement properties of functional walk tests used in the cardiorespiratory domain. Chest 2001;119:256-70.

39. ATS Committee on Proficiency Standards for Clinical Pulmonary Function Laboratories. ATS statement: guidelines for the six-minute walk test. Am J Respir Crit Care Med 2002;166:111-17.

40. Ware JJr, Kosinski M, Keller SD. A 12-Item Short-Form Health Survey: construction of scales and preliminary tests of reliability and validity. Med Care 1996:34:220-33.

41. Bjelland I, Dahl AA, Haug TT, et al. The validity of the hospital anxiety and depression scale. An updated literature review. J Psychosom Res 2002;52:69-77.

42. Snaith RP. The hospital anxiety and depression scale. Health Qual Life Outcomes 2003;1:29.

43. Oldridge N, Hofer S, McGee H, et al. The HeartQoL: Part I. Development of a new core health-related quality of life questionnaire for patients with ischemic heart disease. Eur J Prev Cardiol 2014;21:90-7.

44. Sattelmayer M, Lorenz T, Roder C, et al. Predictive value of the acute low back pain screening questionnaire and the orebro musculoskeletal pain screening questionnaire for persisting problems. Eur Spine J 2012;(21Suppl 6):S773-84.

45. Rikli RE, Jones CJ. Development and validation of criterionreferenced clinically relevant fitness standards for maintaining physical independence in later years. Gerontologist 2013;53: $255-67$.

46. Ada L, Mackey F, Heard R, et al. Stroke rehabilitation: Does the therapy area provide a physical challenge?. Aust J Physiother 1999;45:33-8.

47. Smets EM, Garssen B, Bonke B, et al. The Multidimensional Fatigue Inventory (MFI) psychometric qualities of an instrument to assess fatigue. J Psychosom Res 1995;39:315-25.

48. Smets EM, Garssen B, Cull A, et al. Application of the multidimensional fatigue inventory (MFI-20) in cancer patients receiving radiotherapy. Br J Cancer 1996;73:241-5.

49. Schwartz JE, Jandorf L, Krupp LB. The measurement of fatigue: a new instrument. J Psychosom Res 1993;37:753-62.

50. Watt T, Groenvold M, Bjorner JB, et al. Fatigue in the Danish general population. Influence of sociodemographic factors and disease. J Epidemiol Community Health 2000;54:827-33. 
51. Hagstromer M, Oja P, Sjostrom M. The International Physical Activity Questionnaire (IPAQ): a study of concurrent and construct validity. Public Health Nutr 2006;9:755-62.

52. Broadbent $\mathrm{E}$, Petrie KJ, Main J, et al. The brief illness perception questionnaire. J Psychosom Res 2006;60:631-7.

53. Drummond M. ed. Methods for the economic evaluation of health care programmes. 3rd edn. Oxford: Oxford University Press, 2005

54. Sedgwick P. Simple linaer regression. BMJ 2013. 346 doi: http://dx. doi.org/10.1136/bmj.f2340 (Published 12 Apr 2013)

55. Savović J, Jones HE, Altman DG, et al. Influence of reported study design characteristics on intervention effect estimates from randomized, controlled trials. Ann Intern Med 2012;157:429-38.

56. Wood L, Egger M, Gluud LL, et al. Empirical evidence of bias in treatment effect estimates in controlled trials with different interventions and outcomes: meta-epidemiological study. BMJ 2008;336:601-5.

57. Oldridge N, Hofer S, McGee H, et al. The HeartQoL: Part II. Validation of a new core health-related quality of life questionnaire for patients with ischemic heart disease. Eur J Prev Cardiol 2014;21:98-106.

58. Buysse DJ, Reynolds CF, III, Monk TH, et al. The Pittsburgh sleep quality index: a new instrument for psychiatric practice and research. Psychiatry Res 1989;28:193-213. 